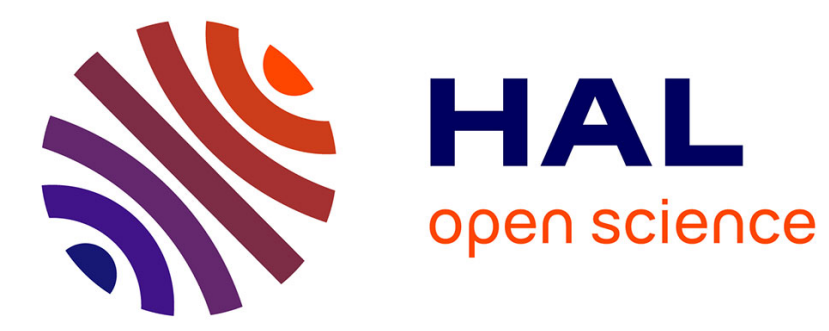

\title{
Geometric Compression for Interactive Transmission
}

Olivier Devillers, Pierre-Marie Gandoin

\section{To cite this version:}

Olivier Devillers, Pierre-Marie Gandoin. Geometric Compression for Interactive Transmission. [Research Report] RR-3910, INRIA. 2000, pp.23. inria-00072743

\section{HAL Id: inria-00072743 \\ https://hal.inria.fr/inria-00072743}

Submitted on 24 May 2006

HAL is a multi-disciplinary open access archive for the deposit and dissemination of scientific research documents, whether they are published or not. The documents may come from teaching and research institutions in France or abroad, or from public or private research centers.
L'archive ouverte pluridisciplinaire HAL, est destinée au dépôt et à la diffusion de documents scientifiques de niveau recherche, publiés ou non, émanant des établissements d'enseignement et de recherche français ou étrangers, des laboratoires publics ou privés. 


\title{
Geometric Compression for Interactive Transmission
}

\author{
Olivier Devillers — Pierre-Marie Gandoin
}

$\mathbf{N}^{\circ} 3910$

Mars 2000

THÈME 2 



\title{
Geometric Compression for Interactive Transmission
}

\author{
Olivier Devillers, Pierre-Marie Gandoin \\ Thème 2 - Génie logiciel \\ et calcul symbolique \\ Projet Prisme \\ Rapport de recherche $\mathrm{n}^{\circ} 3910$ - Mars $2000-23$ pages
}

\begin{abstract}
The compression of geometric structures is a relatively new field of data compression. Since about 1995, several articles have dealt with the coding of meshes, using for most of them the following approach: the vertices of the mesh are coded in an order that partially contains the topology of the mesh. In the same time, some simple rules attempt to predict the position of each vertex from the positions of its neighbors that have been previously coded.

In this article, we describe a compression algorithm whose principle is completely different: the coding order of the vertices is used to compress their coordinates, and then the topology of the mesh is reconstructed from the vertices. This algorithm achieves compression ratios that are slightly better than those of the currently available algorithms, and moreover, it allows progressive and interactive transmission of the meshes.
\end{abstract}

Key-words: geometry, compression, coding, interactivity, mesh, reconstruction, terrain models 


\section{Compression géométrique pour une transmission interactive}

Résumé : La compression de structures géométriques est un domaine relativement récent de la compression de données. Depuis 1995, plusieurs articles ont traité le problème du codage optimal de maillages, en utilisant le plus souvent l'approche suivante: les sommets du maillage sont codés dans un ordre établi pour contenir partiellement la topologie (ou connectivité) du maillage. Parallèlement, un ensemble de règles simples permet de prédire la position du sommet courant à partir des positions de ses voisins qui ont déjà été codés.

Dans cet article, nous décrivons un algorithme de compression dont le principe est complètement différent: l'ordre des sommets est exploité pour comprimer leurs coordonnées, et la topologie est ensuite reconstruite à partir des sommets. Cet algorithme permet d'atteindre des facteurs de compression légèrement supérieurs à ceux des al-

gorithmes actuellement disponibles pour la compression géométrique, et en outre, il permet une transmission progressive et interactive des maillages.

Mots-clés : géométrie, compression, codage, interactivité, maillage, reconstruction, modèles de terrain 


\section{Introduction}

\subsection{Motivations}

In the context of image visualization in a network application, a remote server has to transmit data to a client. These data are usually bitmap data and are transferred through some compression algorithm. This method has also been used in the past for computer graphics images, but in that special case, another solution consists in transmitting the scene description and in running the image synthesis program on the client. A 3D geometric scene is made of polygons, and so is typically coded as a sequence of numbers (the vertices coordinates) and tuples of vertices pointers (the edges joining the vertices).

If the problem of bitmap image compression has already been widely studied, the compression of geometric data, lying between computational geometry and standard data compression, is quite a new field of research.

Yet the rapid growth of image synthesis applications makes necessary the manipulation and the exchange of this type of data in a fast and economical manner. In particular, the numerous possibilities given by the World Wide Web in the field of virtual reality could be dramatically restricted without a fast access to the data. This implies - especially for remote access through low bandwidth lines — that the geometric data would be efficiently structured.

\subsection{Related work}

Among the works dealing with mesh compression, we focus on the following three articles, according to historical and efficiency criterions.

Geometric Compression Through Topological Surgery, by Taubin and Rossignac [9] describes one of the first algorithms that use the transmission order of the mesh vertices to code the topology, and code the vertices positions efficiently by applying prediction schemes. This algorithm - which handles triangle meshes only - decomposes the mesh in triangle strips, and codes the vertices in their order of appearance in the strips, which amounts to code the connectivity of the triangulation. On the other hand, since this order preserves the geometrical neighborhood of the vertices, it allows to linearly predict the position of a vertex from the positions of vertices immediately preceeding in the code. So, instead of coding the absolute coordinates of each vertex, the algorithm uses standard entropy coding methods to send only the error resulting from the predictive technique. Compared to the other methods based on the decomposition of the mesh into triangle strips (in particular, those of Deering

$\mathrm{RR} \mathrm{n}^{\circ} 3910$ 
[7] and Chow [5]), this one seems to give the best compression ratio on practical examples.

Triangle Mesh Compression, by Touma and Gotsman [10] describes another algorithm, whose general principle is quite close. The first difference is the way to traverse the triangulation. The algorithm maintains a list of vertices forming a polygon which contains the coded triangles. This polygon grows by conquering, for each vertex on its boundary, the outside incident triangles. This gives an order over the vertices of the mesh which allows to reconstruct its topology with few additional information. The second difference with the previous algorithm is the method used to predict a vertex position from its predecessors in the code. Besides a linear prediction technique, the algorithm estimates the crease between the current triangles from the previous creases. This yields better compression ratios in practice.

Progressive Compression Of Arbitrary Triangular Meshes, by Cohen-Or, Levin and Remez [6] proposes a method using multiresolution decomposition to achieve progressive compression of the meshes. An initial simplified mesh is refined by insertion of vertices in patches. The position of each new vertex is predicted from the positions of the vertices of the patch where it is inserted, then corrected by additional information. The algorithm uses an efficient scheme of coloring to allow the decoder to recognize the patches. Concerning progressive geometric coding, this algorithm gives the best results published so far, since it yields a code size increase of only $10 \%$ compared to Touma and Gotsman's results.

These algorithms are designed for triangulated surfaces in the 3-dimensional space. The case of genus greater than 0 is deduced from the null genus case by adding some artificial data. It is also important to note that these algorithms first quantize the vertices coordinates to a number of bits typically lying between 8 and 12 .

\subsection{Overview}

In this article, we tackle the problem of coding geometric structures in a different way. We use the fact that in many cases, the 3D objects are constructed automatically from points samples. Hence the topology of a mesh can often be reconstructed from its vertices.

Consequently, our algorithm exploits the transmission order of the vertices to code only their coordinates (description, analysis and features in Sections 2, 3 and 4). Some additional gain can be achieved by using prediction and entropy coding (Section 5). In this form, the algorithm can be applied to any geometric structure as long as a reconstruction algorithm is available for the topology of the original model. 
However, to handle cases where automatic reconstruction is not suitable, we propose also a progressive topological coder (Section 6). Finally, in order to compare our method to previous works, we present experimental results (Section 7).

\section{Description of the algorithm}

In order to simplify the description of the algorithm, we start by handling the onedimensional case. We will see that the generalization to any dimension is straightforward.

We first describe the coding part of the algorithm. Let $S$ be a set of $n$ points lying on a line segment, between 0 and $2^{b}$ (the coordinates of the points are integers coded on $b$ bits). The algorithm begins to code the total number of points on an arbitrary fixed number of bits (32 for example). Then it starts up the main loop which consists in subdividing the current segment in two halves and in coding the number of points contained in one of them (the left half-segment for example) on an optimal number of bits: if the current segment contains $p$ points, the number of points in the half-segment will be coded on $\log _{2}(p+1)$ bits. We will see in Section 5 how it is possible to code a symbol on a fractional number of bits.

So the algorithm maintains a list of segments composed of:

- the segment coordinates,

- the list of all points lying on the segment.

The pseudo-code below details the functioning of the coding part.

$\mathrm{RR} \mathrm{n}^{\circ} 3910$ 
Algorithm Coding of points on a line segment

1. $\mathcal{L} \leftarrow$ original line segment $\mathcal{S}_{0}$

2. output the number of points on $\mathcal{S}_{0}$ on 32 bits

3. while $\mathcal{L}$ not empty

4. do

5. $\quad \mathcal{S} \leftarrow$ pop first segment in $\mathcal{L}$

6. $\quad n \leftarrow$ number of points on $\mathcal{S}$

7. $\quad \mathcal{S}_{1} \leftarrow$ left half of $\mathcal{S}$

8. $\quad n_{1} \leftarrow$ number of points on $\mathcal{S}_{1}$

9. $\quad \mathcal{S}_{2} \leftarrow$ right half of $\mathcal{S}$

10. $n_{2} \leftarrow$ number of points on $\mathcal{S}_{2}$

11. output $n_{1}$ on $\log _{2}(n+1)$ bits

12. if $n_{1}>0$ and length $\left(S_{1}\right)>1$

13. then add $\mathcal{S}_{1}$ at the end of $\mathcal{L}$

14. if $n_{2}>0$ and length $\left(S_{2}\right)>1$

15. then add $\mathcal{S}_{2}$ at the end of $\mathcal{L}$

The loop stops when there are no more divisible segments in the list, that is to say no segment of length greater than 1 . It is to be noted that the only output of the algorithm are the numbers of points lying on the successive segments. The positions of these points are hidden in the order of the output. Indeed, this order contains an implicit binary tree structure.

The decoding part of the algorithm matches exactly its coding part. A list of segments is maintained, but this time the line segment data structure is composed of:

- the segment coordinates,

- the number of points lying on the segment.

The entire decoding part is detailed below. 


\section{Algorithm Decoding of points on a line segment}

1. read the number of points on the original line segment $\mathcal{S}_{0}$ on 32 bits

2. $\mathcal{L} \leftarrow \mathcal{S}_{0}$

3. while $\mathcal{L}$ contains segments of length greater than 1

4. do

5. $\quad \mathcal{S} \leftarrow$ pop first segment in $\mathcal{L}$

6. $\quad n \leftarrow$ number of points on $\mathcal{S}$

7. $\quad$ read the number of points $n_{1}$ on the left half-segment of $\mathcal{S}$ on $\log _{2}(n+1)$ bits

8. $\quad n_{2} \leftarrow n-n_{1}$

9. $\quad$ if $n_{1}>0$

10. $\quad$ then $\mathcal{S}_{1} \leftarrow$ left half of $\mathcal{S}$

11. $\quad$ add $\mathcal{S}_{1}$ at the end of $\mathcal{L}$

12. if $n_{2}>0$

13. $\quad$ then $\mathcal{S}_{2} \leftarrow$ right half of $\mathcal{S}$

14. add $\mathcal{S}_{2}$ at the end of $\mathcal{L}$

As the algorithm progresses, the data read allow to localize the points with more accuracy. Therefore it is possible to visualize the set of points at intermediate stages of the decoding, by displaying the middle point of each segment $\mathcal{S}_{i}$ of the list. Thus the accuracy over the point coordinates is equal to half the length of the corresponding segment.

To generalize this algorithm to any dimension, let us define a cell as the geometric object containing the points to be coded. In dimensions 1, 2 and 3 , the cells are respectively line segments, rectangles, and rectangular parallelepipeds. The only part of the algorithm that differs from a dimension to another is the subdivision of the cell. In dimension $d$, a cell must be subdivided $d$ times (along each of the $d$ axes). Consequently, an order of subdivision for the cells must be chosen (we will come back to this question in the following) and fixed so that the coder and the decoder can communicate.

Figure 1 represents a two-dimensional example. The numbers of points transmitted by the coder are written with the corresponding number of bits below, and the numbers of points deduced by subtraction are written in parentheses. Figure 2 shows the resulting code.

$\mathrm{RR} \mathrm{n}^{\circ} 3910$ 

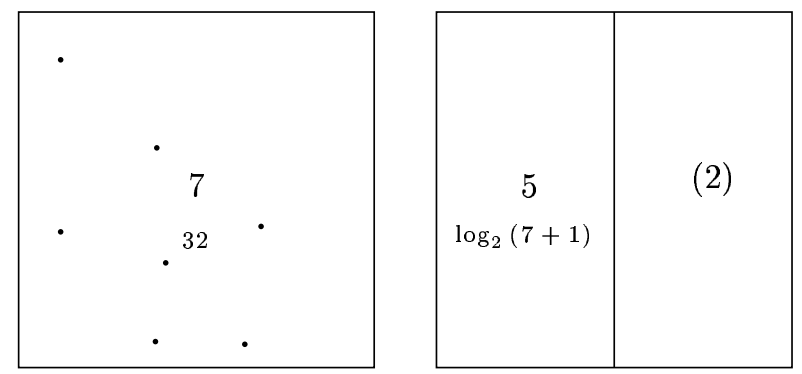

\begin{tabular}{|c|c|}
\hline & \\
2 & 0 \\
$\log _{2}(5+1)$ & $\log _{2}(2+1)$ \\
\hline & \\
\hline$(3)$ & $(2)$ \\
\hline
\end{tabular}
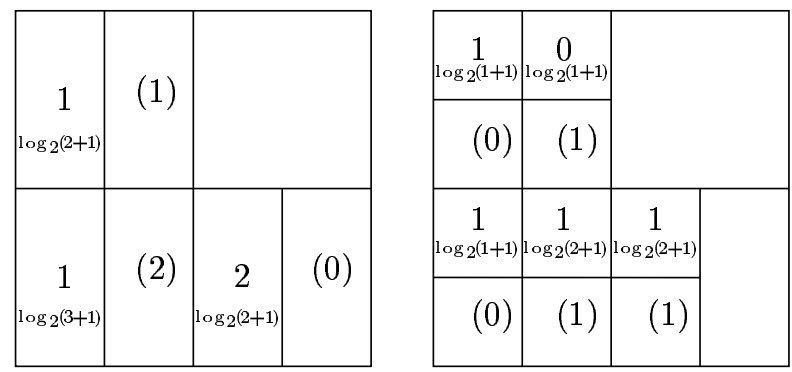

Figure 1: The coding algorithm on a two-dimensional example
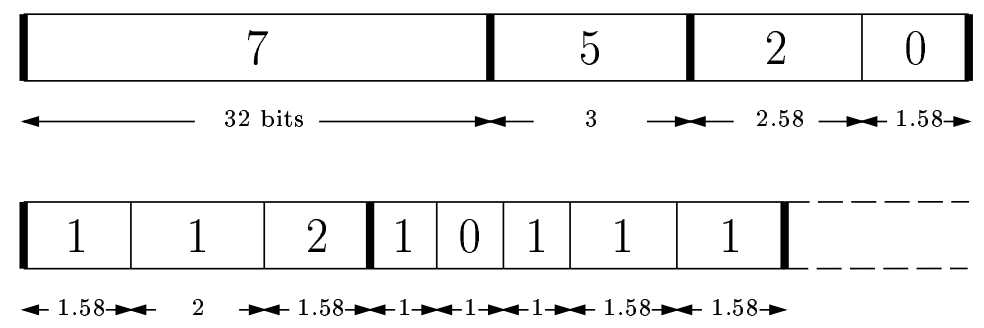

Figure 2: The output code of the two-dimensional example

\section{Theoretical analysis}

\section{1 compression ratio}

To do a theoretical analysis of the algorithm, we will assume that the $n$ points are the vertices of a grid in a $d$-dimensional hypercube. Let $2^{b_{i}}($ for $i=1 . . d$ ) be the side 
lengths of the hypercube (the original cell of the algorithm). In the following, $Q$ will denote the number of bits to code the position of a point: $Q=\sum_{i=1}^{d} b_{i}$.

Let us split up the algorithm in two successive phases:

- separation of the points: the cells are recursively subdivided until each cell of the list contains exactly 1 point,

- final localization: each cell (containing only one point) is subdivided until it reaches the unit size.

Let us calculate the number of bits used to separate the points. With the regularity hypothesis, the dichotomy of a cell containing $c$ points generates two cells containing $c / 2$ points each. Therefore, to separate the $n$ points using this technique, $\left\lceil\log _{2} n\right\rceil$ subdivisions are necessary. If we decompose the algorithm in phases defined by the size of the cells in the current list, the number of cells doubles and the number of points in each cell is reduced by half from a phase to the next one. Now, the number of bits used for the subdivision of a cell containing $c$ points is equal to $\log _{2}(c+1)$. So finally, the total number of bits used to code the separation of the points is given by:

$$
\begin{aligned}
\sum_{i=0}^{\log _{2} n-1} 2^{i} \log _{2}\left(\frac{n}{2^{i}}+1\right)= & -\sum_{i=0}^{\log _{2} n-1} 2^{i} i+\sum_{i=0}^{\log _{2} n-1} 2^{i} \log _{2}\left(n+2^{i}\right) \\
\leq & -\left(n \log _{2}(n)-2 n+2\right) \\
& +\frac{n}{2} \log _{2} \frac{3 n}{2}+\frac{n}{4} \log _{2} \frac{5 n}{4}+\frac{n}{8} \log _{2} \frac{9 n}{8}+\ldots \\
\leq & -n \log _{2} n+2 n+n \log _{2} n\left(\frac{1}{2}+\frac{1}{4}+\frac{1}{8}+\ldots\right) \\
& +n\left(\frac{1}{2} \log _{2} \frac{3}{2}+\frac{1}{4} \log _{2} \frac{5}{4}+\frac{1}{8} \log _{2} \frac{9}{8}+\ldots\right)
\end{aligned}
$$

Finally, the calculations of the sums show that the number of bits used at the end of the separation of the points is less than:

$$
N_{1}=2.402 n
$$

Once a cell contains only one point, it must be subdivided until the point is completely localized. Since $\log _{2} n$ subdivisions have been performed during the separation phase, it remains to subdivide each cell $Q-\log _{2} n$ times. During this phase, a 
subdivision costs 1 bit (the point belongs either to the first half-cell or to the second one). Thus the number of bits used to code the final localization of the points is:

$$
N_{2}=n\left(Q-\log _{2} n\right)
$$

Consequently, the total number of bits used by the algorithm to code the points coordinates is:

$$
N=N_{1}+N_{2}=n\left(Q-\log _{2} n+2.402\right)
$$

If we compare $N$ to $n Q$ (the number of bits used to code the points without compression), we notice that the gain is $\log _{2} n-2.402$ per point, and for the set of points, if we neglect the additive constant, it is $n \log _{2} n$, which corresponds exactly to the order information over the points $\left(\log _{2} n\right.$ bits are necessary to code the index of a point among $n$ ). In other words, the algorithm saves the encoding of the order information over the points.

It is important to observe that this theoretical gain is a lower bound: the regular distribution is the worst-case for the algorithm. In fact, the most non uniform is the distribution, the most efficient is the algorithm. The intuitive idea is that if a cell $c$ is split in two unbalanced parts, then more points share the bit corresponding to $c$, so the global coding of this bit is cheaper. More formally, let $n_{1}, n_{2}, \ldots, n_{2^{j}}$ be the numbers of points in the cells after $j$ subdivision phases, then the cost of the next phase is $\sum_{i=1}^{2^{j}} \log \left(n_{i}+1\right)$, which is maximal when all the $n_{i}$ 's are $n / 2^{j}$, due to the convexity of the log function.

\subsection{Complexity}

The algorithm (compression and decompression) is linear in time and space with respect to $Q n$. However, the time and space constants of the decompression are significantly smaller than the compression ones. Indeed, during the compression, lists of points are stored and must be traversed for each cell subdivision.

\section{Features}

\subsection{Progressivity}

The most interesting feature of the algorithm is the possibility to apply it for progressive coding (and decoding) of the geometric scene. We saw in Section 2 that the only output of the coder were the numbers of points contained in the successive cells, and that the sizes and the positions of those cells were implicitly coded in the order 
of the output. The choice of this order, i.e. of the way to subdivide the original set of points, can be optimized in order to prioritize the progressive coding of the scene. Since the algorithm organizes the cells in a kd-tree, two traversals are possible. The first one is a depth-first traversal: each point is completely localized before the next one is handled. In the second one (breadth-first traversal), all the cells of a same size are processed, generating twice as small cells which will be processed together at the next stage of the algorithm. Therefore after the decoding of an entire batch of cells, it is possible to construct an intermediate version of the set of points such that the precision is the same over each point. A typical manner to do this is to display the center point of each cell of the current list. Of course, if there is no need for an uniform precision over the points, the scene can be visualized at any time of the decompression, and even in real-time.

\subsection{Interactivity}

Thus, for net applications (browsing in particular), it is possible to send successive refined versions of a 3D scene to the final user until he considers that the accuracy is sufficient for his needs. In fact, the method allows to go further in the interactivity with the user. Since the cells are structured in a tree, it is possible, during the decoding, to select one or more subsets of the scene and to refine them and only them. Hence an interactive navigation through a 3D scene, with dilatations and translations, can be optimized from the point of view of the quantity of transmitted information.

\subsection{Lossless compression}

Contrary to the previous works on the subject, our method does not introduce any loss over the original data. The algorithm handles integer coordinates, but the conversion from float does not need any quantization (for typical VRML objects, 24 to 32 bits are necessary to store the coordinates lossless, to be compared with the 8 to 12 bits quantization of $[9,10,6])$. Thus the user can choose to bring the decompression to its end and so obtain the real original object.

\subsection{Dimension}

Another important feature of the algorithm is that it can be applied straightforwardly to data in any dimension. Beyond the three-dimensional space, it can be useful for virtual reality data. Indeed, in the widely spread VRML format, extra data are 
often associated to the vertices, as normal vectors, surfaces, color, radiosity. These data can be handled as additional dimensions and thus compressed exactly like the coordinates. Again, the algorithm performs better with strongly structured data, thus the compression ratio will be smaller when the additional data are correlated to the spatial data (which is the case in usual models).

Moreover, in high dimensions, the choice of the order of subdivision can have important consequences over the compression ratio. If the priority is to obtain intermediate representations of the scene faithful to the original scene, the ideal order is the one of the breadth-first traversal, which consists in subdividing all the cells of a batch along the first dimension, then subdividing the obtained cells along the second dimension, ..., until the dimension $d$, then restarting the same process until the complete localization of the points. But from the point of view of the compression efficiency, the optimal cutting must create empty cells as a priority, and thus, according to the data distribution, it can be more economic to subdivide the cells several times along the same direction. It suffices to add in some header of the compressed data which order of subdivision is best suited to the scene.

\section{Entropy coding and prediction}

The algorithm we described so far is not a compression method in the classical sense of the information theory. Usually, a compression method gives a manner to extract the canonical information of the data (canonical means here non redundant) and to code it. Here, what we have done is a reorganization of the data to drop a part of the information which does not interest us (the order over the points). Therefore, it is natural to think that it remains some redundancy in the information part that we kept (the points coordinates).

\subsection{Arithmetic coding}

Arithmetic coding is a classical entropy coding method developed in the 1980's [8, 11], that allows to code each symbol $s$ of a sequence on $\log _{2} \frac{1}{P}+\epsilon$ bits, where $P$ is the estimated probability of $s$, and $\epsilon$ a small quantity compared to $\log _{2} \frac{1}{P}$. Thus this technique can be quite powerful if coupled to an efficient statistic modeling of the data to be coded.

The first utility of the arithmetic coding for our method is to code the numbers of points of the cells on an optimal number of bits, even if this number is not an integer. Indeed, we saw in the description of the algorithm that for a cell containing $p$ points, 
the number of points in the first half-cell generated by the subdivision was coded on $\log _{2}(p+1)$ bits. In fact, this is possible thanks to the arithmetic coding principle: without a suitable method, this number would be coded on $\left\lceil\log _{2}(p+1)\right\rceil$ bits. Hence the gain for each point would be bounded by $\log _{2} n-3$ instead of $\log _{2} n-2.402$.

\subsection{Prediction methods}

By coding the number of points in the first half-cell generated by the subdivision on $\log _{2}(p+1)$ bits (where $p$ is the number of points in the parent cell), we assume that each integer value lying between 0 and $p$ is equiprobable, with the probability $1 /(p+1)$. To improve the performances of the algorithm, we can try to estimate more precisely the probability of each of those values. To do so, we study the local densities of points in the neighborhood of the cell being subdivided.

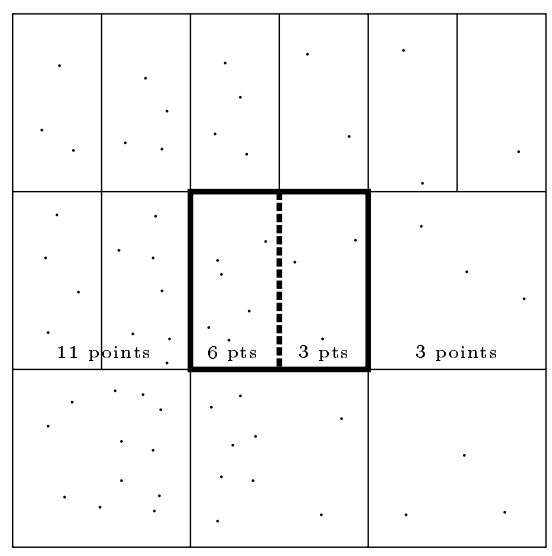

Figure 3: Neighborhood of a two-dimensional cell

The prediction technique relies on the assumption that the local densities in the current cell are correlated with the local densities in its neighborhood. Thus the algorithm analyses the context taking into account all the available information at this precise time of the coding or decoding. Let us give an example to explain the general principle of the method. Let us assume that we have to subdivide vertically the central cell of the Figure 3. The figure shows the state of the kd-tree of cells at this stage. A very simple manner to determine the most probable repartition of the points in the two half-cells is to calculate the percentage of points in the left neighbor cell with respect to the total number of neighbor points (in the left and right neighbor 
cells), and then to assume that the half-cells will match this percentage. Here, we count 11 left neighbors over 14 neighbors, which leads to predict 7 points in the left half of the current cell and 2 points in its right half. From that, a basic method consists in estimating the probabilities of the 10 possible values for the left half-cell with a discrete gaussian law centered at 7 . Thus the actual value of the left half-cell (6 points) will have a strong estimated probability, and so will be coded on a small number of bits.

In this example, the prediction uses only the first order neighborhood, but the technique can be enlarged to higher orders, by giving more weight to the nearest neighbor cells. In fact, the order of the analysed context can be optimized to achieve a satisfying trade-off between the accuracy of the prediction and the algorithm complexity.

With our setting of the parameters, this prediction method provides an additional gain of about $5 \%$ on average, the best results being achieved for $3 \mathrm{D}$ models whose local densities are the most various. It is to be noted that the simple list of cells used in Section 2 is no longer sufficient, since the prediction needs a suitable data structure for a quick access to the cell neighborhood.

\section{Topology coding}

We saw in Section 1.2 that the previous methods of geometric compression needed the topology of the object to achieve some prediction on the position of the points and so manage compression of these data. The original point in our method is that we obtain similar results (see Section 7.2) without the support of the topological information. That is why this method is particularly well-suited for models whose topology can be automatically reconstructed.

However, for many $3 \mathrm{D}$ objects, this automatic reconstruction cannot be done in a lossless way, so we propose an algorithm that progressively codes the topology of a geometric structure. This algorithm relies on the same principle of cells subdivision used by the positions coder, so can be applied to the object simultaneously.

\subsection{Description of the algorithm}

Our algorithm codes the minimal connectivity information of the structure in the sense that only the object edges are coded. Consequently, some extra work is necessary to reconstruct the facets of the mesh and the adjacency relations between them when the final application needs these informations. In the following, we will call 
super-edge an edge between 2 cells. We say that there is a super-edge between 2 cells when there is an edge joining a point of one cell to a point of the other. We call neighbors 2 cells joined by a super-edge.

The algorithm codes these super-edges. Each cell stores a list of its neighbors (at the beginning, the first cell, which is the bounding box of the object, has no neighbor). For each subdivision, we code how the super-edges incident to the original cell are connected to its 2 sub-cells. More precisely, there are 3 possibilities for each super-edge $E$ incident to the original cell $C$ :

- $E$ is incident to the first sub-cell $c_{0}$,

- $E$ is incident to the second sub-cell $c_{1}$,

- $E$ is incident to $c_{0}$ and $c_{1}$.

Consequently, for each cell subdivision, each super-edge generates a code on $\log _{2}(3)$ bits. Besides, one additional bit is necessary to code the possible appearance of a new super-edge between the sub-cells $c_{0}$ and $c_{1}$. Of course, when a cell subdivision generates an empty cell, there is no topological information to be transmitted. The non empty sub-cell inherits all the super-edges of its parent cell.

Regarding the visualization of intermediate versions of the original model, some post-processing regularization work must be done, since the early stages of the decompression can create edge crossings (see Figure 4). It is only during the final localization, when each cell contains one point, that the topology of the intermediate object has the same properties as the original one.

Figure 4 shows a two-dimensional example (including the original mesh). The code of Figure 5 corresponds to a clockwise description of the super-edges, starting with the bottom-left cell.

\subsection{Theoretical analysis}

The amount of memory used by this method to code the topology of a geometric structure depends on the average degree of a cell. Indeed, the subdivision of a cell having $d$ neighbors costs exactly $d \log _{2}(3)+1$ bits (if it is a "true" subdivision, i.e. if it does not generate an empty cell). Now, it takes exactly $n-1$ "true" subdivisions to separate the $n$ points of an object, i.e. to code its whole topology. Consequently, if the average degree of a cell is $d$, the progressive coding of the topology takes less than $d \log _{2}(3)+1$ bits per vertex.

The difficulty is to evaluate this average degree, which must not be confused with the average degree of the original geometric object: it is the average number 

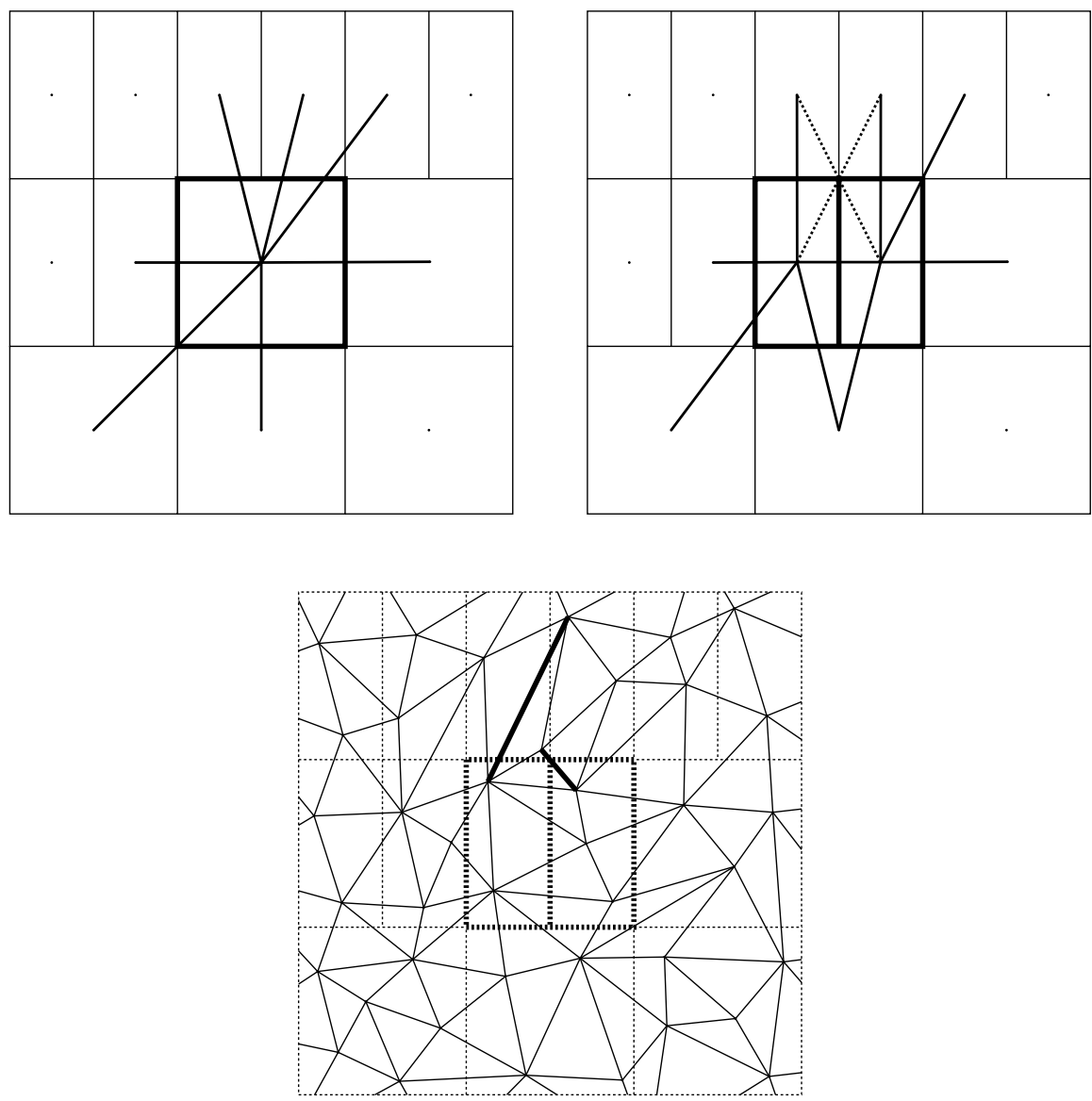

Figure 4: Coding the topology on a two-dimensional example

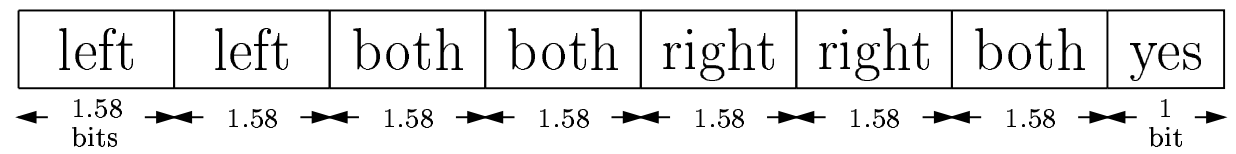

Figure 5: The output code of the two-dimensional example

of neighbors of a cell during the separation phase of the algorithm, therefore, it is slightly greater than the average degree of a vertex. Typically, for a triangular mesh, 
the average degree of a cell is about 7, which corresponds to a cost of 12 bits per vertex for the topology coding.

\subsection{Future work}

\subsubsection{Prediction}

As it is, the compression achieved by this topology coding algorithm is far from the results of [10] or [6]. However, this method should allow us to handle special connectivity cases, like triangular meshes, in a much more efficient way using prediction and entropy coding. For example, the appearance of a new super-edge between the sub-cells is highly probable and can be coded efficiently using entropy coding. Moreover, the connectivity of a parent cell neighbors to the two sub-cells can often be predicted from various informations like cells positions, sizes, or number of points. This work will be described in a forthcoming paper.

\subsubsection{Automatic reconstruction}

An example of application where the topology can be reconstructed from the set of points is given by terrain models. In most cases, the connectivity of such models is obtained by computing the Delaunay triangulation of the 2D-projected set of points. In some cases however, slight variations are introduced in the object topology (in order to follow roads or rivers for instance). In such cases, 5 to $10 \%$ only of the edges are not Delaunay edges. It suffices to code them, then to compute the constrained Delaunay triangulation to reconstruct the model topology. In 3D, the same technique can be applied to tetrahedral meshes. More generally, the topology coder allows to "help" any reconstruction algorithm by coding a subset of the object edges.

\section{$7 \quad$ Experimental results}

\section{$7.1 \quad$ Terrain models}

Figure 6 gives results of our method applied to some terrain models. The first two lines come from a GIS database covering the region of Vancouver, whereas the third one corresponds to a simple terrain model composed of 14641 vertices with 12/12/10 bits coordinates. This example allows to visualize the progression of the decoding on Figures 9 to 12.

$\mathrm{RR} \mathrm{n}^{\circ} 3910$ 


\begin{tabular}{|c|c|c|c|c|c|}
\hline & $\begin{array}{c}\text { number of } \\
\text { vertices }\end{array}$ & $\begin{array}{c}\text { source } \\
\text { data }\end{array}$ & $\begin{array}{c}\text { comp. } \\
\text { data }\end{array}$ & $\begin{array}{c}\text { comp. } \\
\text { ratio }\end{array}$ & $\begin{array}{c}\text { theor. } \\
\text { ratio }\end{array}$ \\
\hline \hline rivers & 120998 & 650365 & 341365 & $52 \%$ & $66 \%$ \\
& & 43 & 22.6 & & \\
\hline vancouver & 908907 & 4885376 & 2169750 & $44 \%$ & $60 \%$ \\
& & 43 & 19.1 & & \\
\hline terrain & 14641 & 62225 & 33776 & $54 \%$ & $66 \%$ \\
& & 34 & 18.5 & & \\
\hline
\end{tabular}

Figure 6: results of the compression on terrain models (in the columns 3 and 4 , we give the size of the data in bytes and the corresponding number of bits per vertex)

\section{2 $\quad$ Standard $3 \mathrm{D}$ objects}

We present in Figure 7 some results of the method described in this paper compared to those of Taubin and Rossignac [9] and Touma and Gotsman [10]. We saw in Section 1.2 that these two methods coded the connectivity and the coordinates of the mesh vertices. However, the figures that appear here concern only the coding of the coordinates, so are comparable to the results of our algorithm. The columns 4 and 5 have been extracted from the article of Touma and Gotsman, and we have applied our method to the same geometric models to obtain the column 6 . It is to be noted that the coordinates of these 3D models have been prior quantized on 8 bits to follow exactly the same process as in the first two cited articles. The differences of efficiency come from the structure of the models: "eight" and "shape" have a very regular structure which suits the predictive method of Touma and Gotsman. On the contrary, "dumptruck" and "engine" are more complex models containing variations of local density more suitable for our algorithm. Regarding the method of CohenOr et al. [6], we do not have results for the same models, but the authors report an average code size increase of about $10 \%$ compared to the Touma and Gotsman results.

In Figure 8, we show what can be obtained by automatic reconstruction [3] from the set of points, without any connectivity information. 


\begin{tabular}{|c|c|c|c|c|c|}
\hline & $\begin{array}{c}\text { number of } \\
\text { vertices }\end{array}$ & $\begin{array}{c}\text { source } \\
\text { data }\end{array}$ & $\begin{array}{c}\text { IBM } \\
1996\end{array}$ & $\begin{array}{c}\text { G \& T } \\
1998\end{array}$ & $\begin{array}{c}\text { our } \\
\text { algo. }\end{array}$ \\
\hline \hline beethoven & 2655 & 7965 & 4982 & 3576 & 3201 \\
& & 24 & 15.0 & 10.8 & 9.6 \\
\hline blob & 8036 & 24108 & 10352 & 7951 & 8937 \\
& & 24 & 10.3 & 7.9 & 8.9 \\
\hline cow & 3066 & 8815 & 4878 & 3376 & 3419 \\
& & 23 & 12.7 & 8.8 & 8.9 \\
\hline dumptruck & 11738 & 32280 & 20351 & 11162 & 7218 \\
& & 22 & 13.9 & 7.6 & 4.9 \\
\hline eight & 766 & 2011 & 1146 & 683 & 1018 \\
& & 21 & 12.0 & 7.1 & 10.6 \\
\hline engine & 2164 & 6222 & 4703 & 3425 & 2492 \\
& & 23 & 17.4 & 12.7 & 9.2 \\
\hline shape & 2562 & 7686 & 4578 & 2990 & 4052 \\
& & 24 & 14.3 & 9.3 & 12.7 \\
\hline triceratops & 2832 & 7788 & 3673 & 2937 & 2843 \\
& & 22 & 10.4 & 8.3 & 8.0 \\
\hline \hline total & 33819 & 96875 & 54663 & 36100 & 33180 \\
& & 22.9 & 12.9 & 8.5 & 7.8 \\
\hline
\end{tabular}

Figure 7: benchmark on the compression of 3D models

\section{Conclusion}

We have presented a new method of geometric compression whose originality is to perform coordinates compression without the support of the connectivity information. Besides achieving slightly better ratios than the current available algorithms, this technique allows lossless coding and interactive decoding. Moreover, the algorithm is valid for any dimension, and simple to implement. This method is particularly well-suited to any geometric structure whose topology is reconstructible from its vertices, which is the case of terrain models. However, the progressive topology coding feature permits to handle any geometric structure (not only triangular meshes). Concerning the special case of triangular meshes, a future publication will show how prediction and entropy coding can lead to competitive compression ratios for the connectivity. 


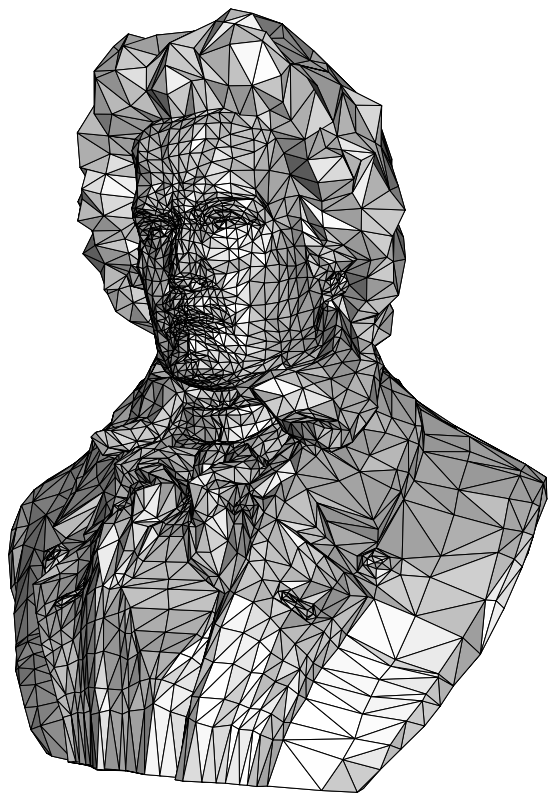

(a) Original topology

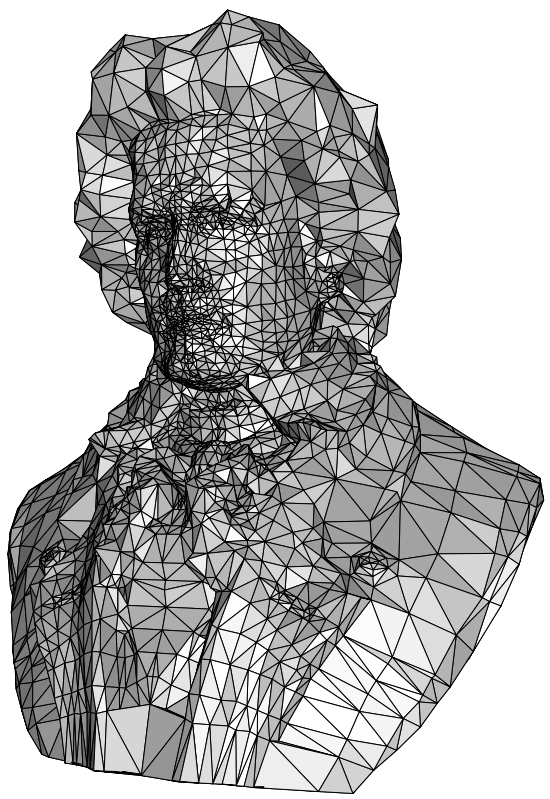

(b) Reconstructed topology

Figure 8: Beethoven (geometry cost: 9.6 bits per vertex)

On the other hand, future works will consider the use of some 3D surface reconstruction methods $[1,2,4,3]$, which give a necessary and sufficient condition over the object sample to guaranty a valid reconstruction. Thus, by possibly adding a small number of points (or edges, using the topology coder) to the original set, it will be possible to reconstruct the object topology.

Another important perspective is to improve the predictive scheme, whose current results are not completely satisfying. It could be achieved by a more precise analysis of the points distribution in the neighborhood of the current cell, and by applying optimization methods for the parameters setting.

\section{References}

[1] N. Amenta and M. Bern. Surface reconstruction by Voronoi filtering. In Proc. $A C M$ SoCG, pages 39-48, 1998. 
[2] Fausto Bernardini and Chandrajit L. Bajaj. Sampling and reconstructing manifolds using alpha-shapes. In Proc. 9th Canad. Conf. Comput. Geom., pages 193-198, 1997.

[3] J.-D. Boissonnat and F. Cazals. Smooth shape reconstruction. In Proc. ACM So $C G, 2000$. (to appear).

[4] J.-D. Boissonnat and B. Geiger. 3-dimensional reconstruction of complex shapes based on the Delaunay triangulation. In Biomedical Image Processing and Biomedical Visualization, volume 1905, pages 964-975, 1993.

[5] M. Chow. Optimized geometry compression for real-time rendering. In Proc. IEEE Visualization, pages 347-354, 1997.

[6] D. Cohen-Or, D. Levin, and O. Remez. Progressive compression of arbitrary triangular meshes. In Proc. IEEE Visualization, pages 67-72, 1999.

[7] M. Deering. Geometry compression. In Proc. SIGGRAPH, pages 13-20, 1995.

[8] J. Rissanen and G. G. Langdon. Arithmetic coding. IBM J. Res. Develop., 23(2):149-162, 1979.

[9] G. Taubin and J. Rossignac. Geometric compression through topological surgery. Research Report RC-20340, IBM Research Division, 1996.

[10] C. Touma and C. Gotsman. Triangle mesh compression. In Proc. Graphics Interface, pages 26-34, 1998.

[11] I.H. Witten, R. Neal, and J.G. Cleary. Arithmetic coding for data compression. Communications of the ACM, 30(6):520-540, 1987. 


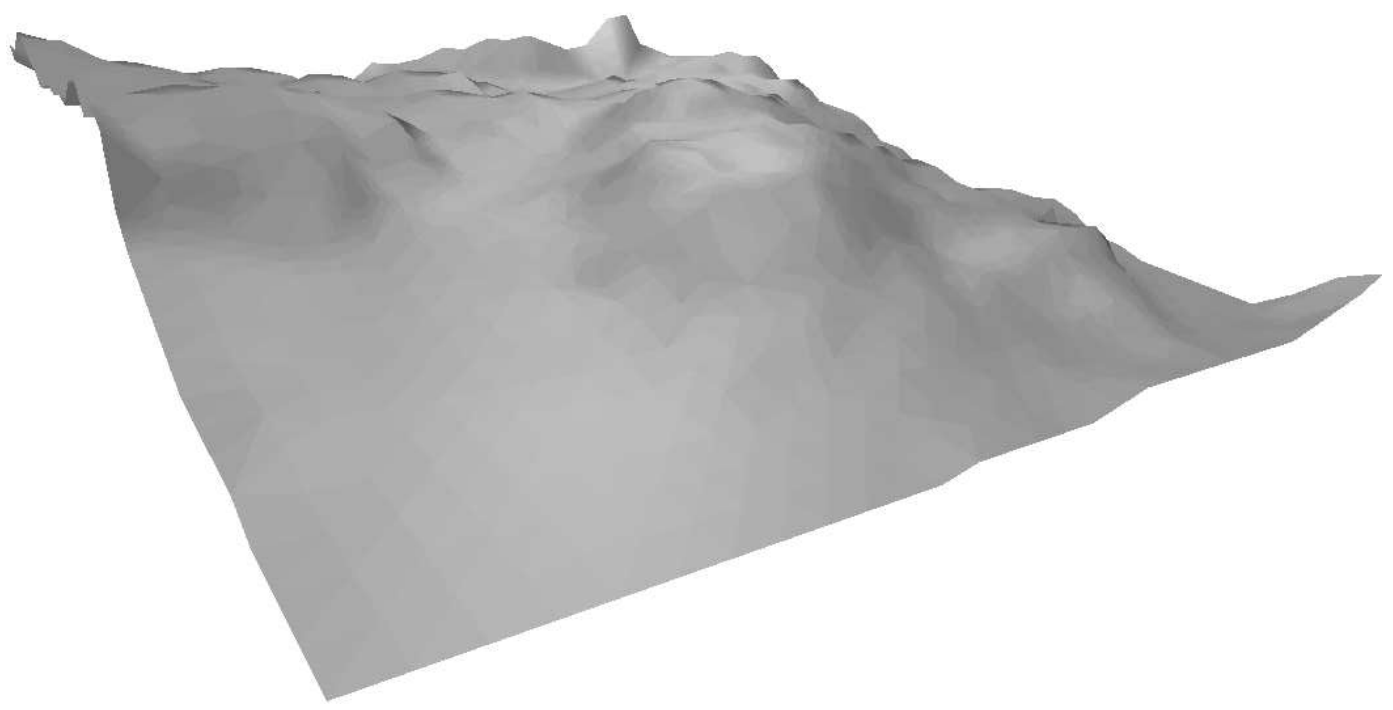

Figure 9: precision $=5$ bits, compression ratio $=3 \%$

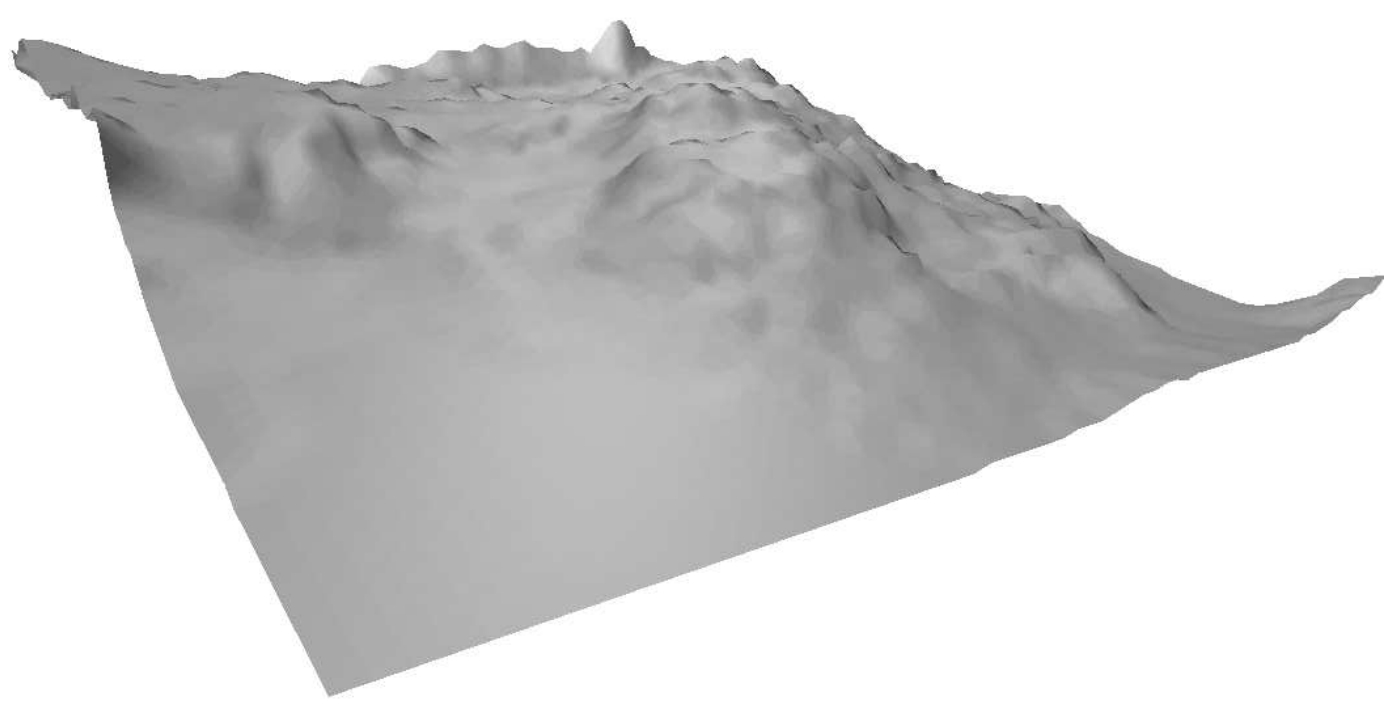

Figure 10: precision $=6$ bits, compression ratio $=8 \%$ 


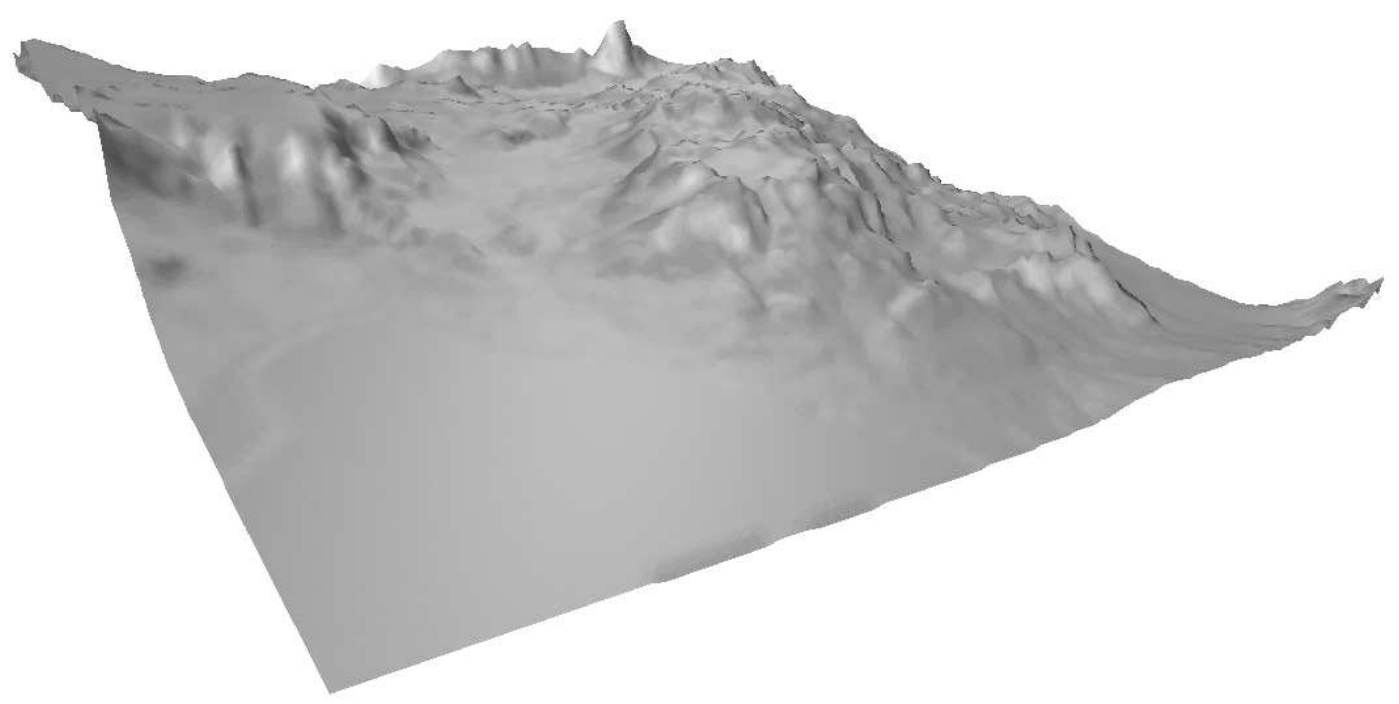

Figure 11: precision $=7$ bits, compression ratio $=16 \%$

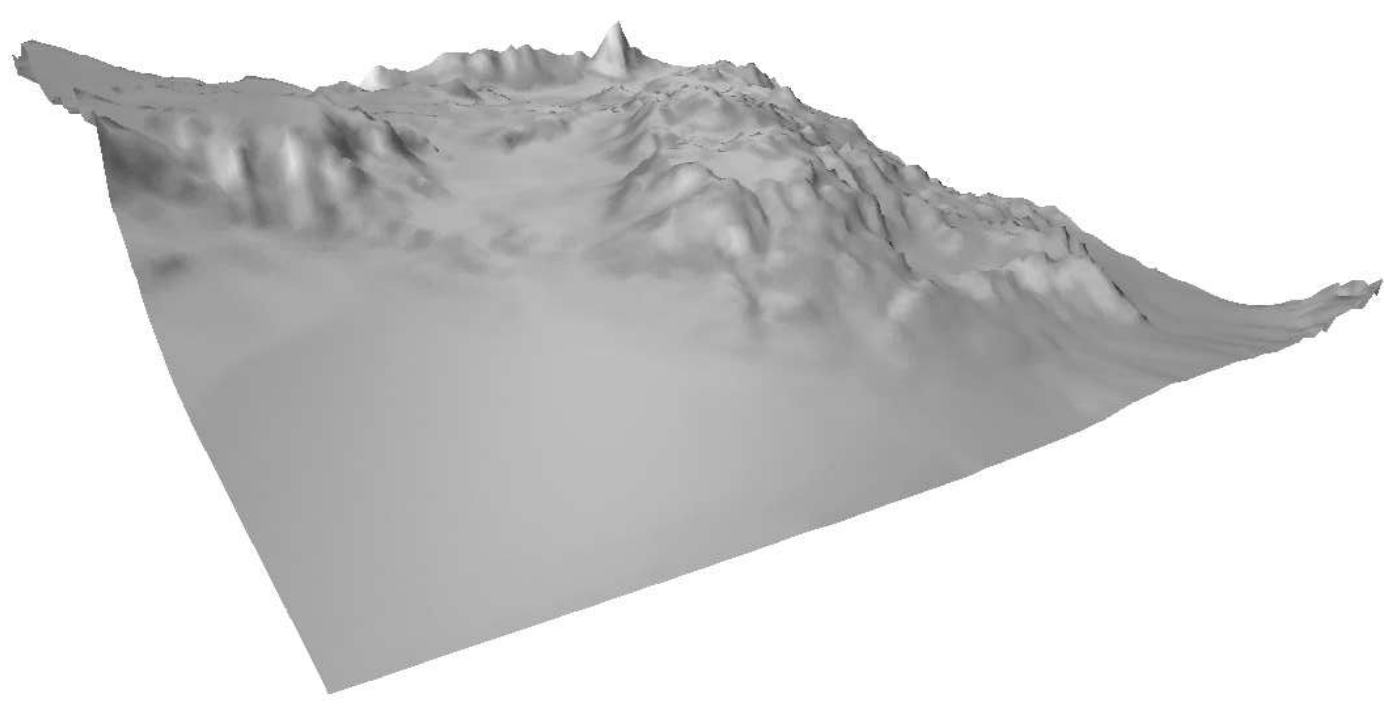

Figure 12: precision $=12$ bits (lossless compression), c. r. $=54 \%$ 


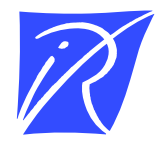

Unité de recherche INRIA Sophia Antipolis 2004, route des Lucioles - B.P. 93 - 06902 Sophia Antipolis Cedex (France)

Unité de recherche INRIA Lorraine : Technopôle de Nancy-Brabois - Campus scientifique 615, rue du Jardin Botanique - B.P. 101 - 54602 Villers lès Nancy Cedex (France)

Unité de recherche INRIA Rennes : IRISA, Campus universitaire de Beaulieu - 35042 Rennes Cedex (France)

Unité de recherche INRIA Rhône-Alpes : 655, avenue de l'Europe - 38330 Montbonnot St Martin (France)

Unité de recherche INRIA Rocquencourt : Domaine de Voluceau - Rocquencourt - B.P. 105 - 78153 Le Chesnay Cedex (France)

Éditeur

INRIA - Domaine de Voluceau - Rocquencourt, B.P. 105 - 78153 Le Chesnay Cedex (France)

http://www.inria.fr

ISSN 0249-6399 